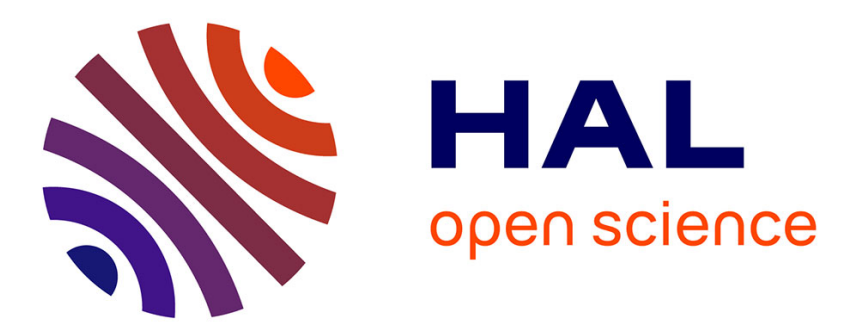

\title{
On two dissimilarity-based measures of functional beta diversity
}

\author{
Carlo Ricotta, Evsey Kosman, Marco Caccianiga, Bruno E L Cerabolini, \\ Sandrine Pavoine
}

\section{- To cite this version:}

Carlo Ricotta, Evsey Kosman, Marco Caccianiga, Bruno E L Cerabolini, Sandrine Pavoine. On two dissimilarity-based measures of functional beta diversity. Ecological Informatics, 2021, 66, pp.101458. 10.1016/j.ecoinf.2021.101458 . hal-03463587

\section{HAL Id: hal-03463587 \\ https://hal.science/hal-03463587}

Submitted on 2 Dec 2021

HAL is a multi-disciplinary open access archive for the deposit and dissemination of scientific research documents, whether they are published or not. The documents may come from teaching and research institutions in France or abroad, or from public or private research centers.
L'archive ouverte pluridisciplinaire HAL, est destinée au dépôt et à la diffusion de documents scientifiques de niveau recherche, publiés ou non, émanant des établissements d'enseignement et de recherche français ou étrangers, des laboratoires publics ou privés. 


\title{
On two dissimilarity-based measures of functional beta diversity
} \\ ${ }^{1}$ Department of Environmental Biology, University of Rome 'La Sapienza', Rome, Italy; ${ }^{2}$ Institute for Cereal Crops \\ Improvement, Tel Aviv University, Tel Aviv, Israel; ${ }^{3}$ Department of Biosciences, University of Milano, Milano, Italy; \\ ${ }^{4}$ Department of Biotechnology and Life Sciences, University of Insubria, Varese, Italy; ${ }^{5}$ Centre d'Ecologie et des \\ Sciences de la Conservation (CESCO), Muséum National d'Histoire Naturelle, CNRS, Sorbonne Université, Paris, \\ France. \\ *Corresponding author. E-mail: carlo.ricotta@uniroma1.it
}

Abstract. In this paper, we propose two related versions of a dissimilarity-based measure of functional beta diversity, together with the associated tests for differences in beta diversity among different groups of samples. Both measures are based on the optimal functional matching between the species in two samples. As such, they are tightly connected to Hurlbert's seminal work on encounter-based diversity measures. The behavior of the proposed measures is illustrated with one worked example on the functional turnover of Alpine species along a successional gradient. Results show that both measures proved able to detect the functional turnover of vegetation along the chronosequence. The method, for which we provide a simple R function, further allows to evaluate the functional contribution of single sampling units to the overall beta diversity of any kind of species assemblages.

Keywords: Algorithmic measure; Assignment problem; Functional dissimilarity; Optimization process; Randomization test.

\section{Introduction}

Beta diversity measures the variability in species composition among a set of sampling units and is considered to be a key signature of the ecological processes that make species assemblages more or less similar to one another (Anderson et al. 2011; Bennet and Gilbert 2016). Since the pioneering work by Whittaker (1972), there have been intense discussions on how to measure beta diversity and how to test for differences in beta diversity among different groups of samples. For reviews, see e.g. Lande (1996), Koleff et al. (2003), Anderson et al. (2006, 2011), Jost (2007), Tuomisto (2010a, 2010b), Chase et al. (2011), Chao and Chiu (2016), Legendre and De Cáceres (2013), Ricotta (2017), Chao and Ricotta (2019) and references therein.

Irrespective of how beta diversity is measured, an important requisite for diversity measures is their ecological interpretability. According to the seminal paper of Stuart Hurlbert (1971), meaningful diversity indices should have a straightforward biological interpretation: "We therefore can muddle along with a plethora of indices, each supported by at least one person's intuition and a few recommended by fashion, or we can sharpen our thoughts and rephrase our questions in terms of biologically meaningful properties [...]" (Hurlbert 1971 p. 579).

Among these properties, the probability of intra- and interspecific encounters is a variable of interest, as it is directly related to the potential ecological interactions among all individuals and species in the community (Hurlbert 1971; Patil and Taillie 1982). This encounter-based approach is 
even more important for functional diversity where, unlike for classical diversity measures, the species are not considered equally dissimilar from each other. In a sense, dealing with functional diversity measures, the potential amount of ecological interactions among different individuals is ideally related to their functional resemblance.

In this paper, we thus propose two different versions of a dissimilarity-related index of functional beta diversity, together with the associated tests for differences among different groups of samples. Both indices are based on the optimal functional matching between the species in two samples. As such, they are tightly connected to Hurlbert's encounter-based approach.

\section{A dissimilarity-based index of functional beta diversity}

Given a set of $N$ samples, let $p_{j k}$ be the relative abundance of species $j=1,2, \ldots, S$ in sample $k=1,2, \ldots, N$ such that $0 \leq p_{j k} \leq 1$ and $\sum_{j}^{S} p_{j k}=1$. The information on the species functional organization within samples is usually represented by a symmetric $S \times S$ matrix of pairwise functional dissimilarities $d_{i j}$ between species $i$ and $j$ in the range [0,1] (with $d_{i j}=d_{j i}$ and $d_{i i}=0$ ) which represent the multivariate differences in the character states among the $S$ species.

To calculate a dissimilarity-based index of functional beta diversity, the first step consists in calculating the pairwise functional dissimilarity $D_{h k}$ between any pair of samples $h$ and $k$. To this end, Ricotta et al. (2021a) first used an algorithmic measure originally developed by Kosman (1996) and Gregorius et al. (2003) to calculate genetic distances between populations. The measure is based on the optimal matching between the species abundances in $h$ and $k$ so as to minimize the overall functional dissimilarity between both samples.

The dissimilarity index $D_{h k}$ is calculated as follows: given two samples $h$ and $k$, with $n$ individuals in both samples, each individual in $h$ is matched to an individual in $k$ in order to get $n$ pairs that minimize the sum of functional dissimilarities between the individuals in each pair (Kosman \& Leonard, 2007). The pairs are built such that all individuals in both samples are used only once. The overall functional dissimilarity between the two samples is then obtained as the mean dissimilarity between each pair of individuals (i.e. by dividing the sum of functional dissimilarities by the $n$ pairs of individuals). However, since the number of individuals in $h$ and $k$ is generally not the same, to get a complete matching between the samples, this procedure is usually performed on the species relative abundances in both samples. The algorithmic dissimilarity $D_{h k}$ can be thus interpreted as the minimum cost per individual needed to change the character states of the species in sample $h$ to the states of the species in $k$ (Gregorius et al. 2003). 
Finding the optimal matching between the species abundances in $h$ and $k$ is known as the assignment problem, a special type of linear programming or linear optimization problem (Dantzig and Thapa 1997). Dealing with species relative abundances, the functional dissimilarity between samples $h$ and $k$ can be formulated as (Gregorius et al., 2003):

$$
D_{h k}=\min _{\pi} \sum_{i}^{S} \sum_{j}^{S} d_{i j} \times \pi(i, j)
$$

where $\pi(i, j)$ is the relative abundance of species $i$ in sample $h$ that is matched with species $j$ in sample $k$. Since $D_{h k}$ is essentially a mean dissimilarity between matched pairs of individuals, if the functional dissimilarity $d_{i j}$ between each pair of individuals is in the range $[0,1]$, the resulting mean dissimilarity also ranges between 0 and 1. Kosman (2014) further showed that if all species in $h$ and $k$ are considered maximally dissimilar from each other (i.e. if $d_{i j}=1$ for all species $i$ in sample $h$ and species $j$ in sample $k$ ), $D_{h k}$ will be equal to $D_{h k}=\frac{1}{2} \sum_{i, j}^{S}\left|p_{i h}-p_{j k}\right|$.

A simple way to generalize $D_{h k}$ to more than two samples, which is usually adopted in community ecology for calculating the beta diversity of a set of $N$ samples (but see e.g. Diserud and Ødegaard 2007), consists in calculating the mean value of $D_{h k}$ for all possible pairs of samples:

$\beta_{N}=\frac{\sum_{k>h}^{N} D_{h k}}{N(N-1) / 2}$

Once beta diversity has been calculated, the next step is how to test for differences in beta diversity among different groups of samples. To this end, Anderson (2006) proposed a multivariate analogue of Levene's (1960) test, which is directly connected to the way $\beta_{N}$ is calculated. The test can be considered in two steps: first, starting from the functional dissimilarities between all pairs of sampling units $D_{k n}$, the dissimilarity $D_{k}$ of each individual sample from its group centroid in multivariate space is calculated according to McArdle and Anderson (2001). Next, the average of these dissimilarities among groups is compared using ANOVA. A $P$-value can be then obtained with either the traditional tables on $F$-distribution or by using a permutation procedure (Anderson 2006).

A drawback of this method is that the dissimilarity of individual samples from the group centroid depends on the number of samples in each group. Take for example a group composed of five 
$114 D_{\bar{k}}=\frac{\sum_{h \neq k}^{N} D_{h k}}{N-1}$

$$
p_{j \eta}=\frac{\sum_{h \neq k}^{N} p_{j h}}{N-1}
$$

According to this leave-one-out approach, $\eta$ can be interpreted as the compositional centroid of the $N-1$ samples that differ from $k$ in Euclidean space (see Champely and Chessel 2002). A multiple-site measure of beta diversity can be then obtained by taking the mean of the

maximally dissimilar samples, i.e. with $D_{h k}=1$ for all $h \neq k$. In this case, the dissimilarity $D_{k}$. of each individual sample from its group centroid is equal to $D_{k}=0.632$. By contrast, for ten maximally dissimilar samples, $D_{k}=0.671$ (for details, see Anderson 2006). Accordingly, this test works correctly only with fully balanced designs with the same number of samples in each group. dissimilarity of each individual sample $k$ from all other $N-1$ samples in the same group:

The same approach was used by Violle et al. (2017) and Kosman et al. (2019) to calculate the mean distance in trait space of a species to all other species in a community. The main advantage of $D_{\bar{k}}$ over $D_{k}$. is that $D_{\bar{k}}$ is not influenced by the number of samples in each group. Like for the Anderson (2006) test, the average of these dissimilarities among groups can be then compared using standard ANOVA (see the example in Appendix 1).

\section{A second index of beta diversity}

A second method for deriving a measure of multiple-site functional dissimilarity among sampling units may consist in calculating the dissimilarity of Kosman (1996) and Gregorius et al. (2003) $D_{k \eta}$ between the species relative abundances in sample $k$ and the species relative abundances in an hypothetical complementary sample $\eta$. This complementary sample is obtained by pooling together the species relative abundances of all $N-1$ samples that are different from $k$ such that the relative abundance of species $j$ in $\eta$ is calculated as:

$$
\text { dissimilarities } D_{k \eta} \text { over the } N \text { samples: }
$$


$137 \quad \beta_{\eta}=\frac{\sum_{k}^{N} D_{k \eta}}{N}$

138

If beta diversity is calculated according to Eq. 5, a test for differences in beta diversity among different groups of samples can be then performed in the usual way, by comparing the mean values of $D_{k \eta}$ within each group with ANOVA.

\section{Worked example}

\subsection{Data}

To illustrate the behavior of the proposed measures, we used a data set of Alpine vegetation sampled by Caccianiga et al. (2006) along a primary succession at the foreland of the Rutor Glacier (Northern Italy). The data set has been already used in previous studies on community structure and diversity (Ricotta et al., 2016; Ricotta et al., 2020) and is composed of 45 species in 59 plots of approximately $25 \mathrm{~m}^{2}$. All data are available in Ricotta et al. (2016, Appendix S2). The species abundances in each plot were measured with a five-point ordinal scale transformed to ranks. The plots were classified into three successional stages based on the age of the glacial deposits: earlysuccessional stage (17 plots), mid-successional stage (MSS; 32 plots), and late-successional stage (10 plots).

For all 45 species sampled at the three successional stages, we used six quantitative traits that are related to their successional status along the primary succession: canopy height $(\mathrm{CH} ; \mathrm{mm})$, leaf dry mass content (LDMC; \%), leaf dry weight (LDW; mg), specific leaf area (SLA; mm2 $\times$ mg-1), leaf nitrogen content (LNC; \%), and leaf carbon content (LCC; \%). All traits can be found in Caccianiga et al. (2006, Table 2).

First, we used the Euclidean distance to compute a matrix of pairwise functional distances between the 45 species from the six functional traits. For this purpose, all trait values for the 45 species were standardized to zero mean and unit standard deviation. The output functional distances were then scaled in the range $[0,1]$ by dividing each distance by the maximum value in the distance matrix.

Using the algorithmic approach of Kosman (1996) and Gregorius et al. (2003), we next calculated the beta diversity components (i.e. dissimilarities) $D_{\bar{k}}$ and $D_{k \eta}$ for each sample in each successional stage. All calculations were performed with a new R script (available in the electronic Appendix 1 and 2 of this paper) that modifies the $\mathrm{R}$ function dislptransport in Ricotta et al. (2021, Appendix S3). We finally tested for differences in beta diversity among the three successional stages by comparing the average of these dissimilarities among groups with ANOVA. 
$170 \mathrm{P}$-values were obtained by using a permutation procedure. Among the many available permutation

171 procedures in ANOVA designs (Anderson and Ter Braak 2003; Anderson 2004), we used the 172 simplest approach, which consists in permuting individual observation units among the three 173 successional stages of the Rutor chronosequence. To this end, we reshuffled $17+32+10=59$ 174 observed dissimilarities $D_{\bar{k}}$ and $D_{k \eta}$ into random groups of 17, 32, and 10 units, respectively (9999 175 permutations) and recalculated the F-values for each permutation. The same permutation procedure, 176 was then used to perform a post-hoc pairwise t-test with Holm correction of the values of $D_{\bar{k}}$ and $D_{k \eta}$ between the three successional stages.

178

179

180

181

\subsection{Results}

The results of the permutational ANOVA on the values of $D_{\bar{k}}$ and $D_{k \eta}$ among the three successional stages were in both cases highly significant $\left(F\left(D_{\bar{k}}\right)=71.56, p<0.001\right.$ and $\left.F\left(D_{k \eta}\right)=18.91, p<0.001\right)$. For both dissimilarity coefficients $D_{\bar{k}}$ and $D_{k \eta}$, the within-group dispersion (or beta diversity) progressively decreased along the primary succession (Figure 1). As shown by Caccianiga et al. (2006) and Ricotta et al. (2016), the significantly higher beta diversity of the early-successional samples may be due to the random dispersal mechanisms that drive the colonization of the moraine ridges in the first successional stages (abiotic filter). In contrast, the lower beta diversity of the mid- and late successional samples is associated to a lower level of stochasticity in the colonization process of the later successional stages and hence to an increased level of functional homogeneity among different sampling units (biotic filter).

Note that, since $D_{\bar{k}}$ is essentially an average dissimilarity between pair of samples, while $D_{k \eta}$ is the dissimilarity between a given sample $k$ and a complementary sample $\eta$ that is obtained by pooling together the species relative abundances of all samples that are different from $k$, the values of $D_{k \eta}$ are generally lower than the values of $D_{\bar{k}}$ (see e.g. Figure 1$)$.

\section{Discussion}

In this paper we proposed two measures of functional beta diversity, $\beta_{N}$ and $\beta_{\eta}$ which originate from Whittaker's (1972) suggestion that beta can be summarized from a dissimilarity coefficient between pairs of samples (see also Chao and Chiu 2016). The proposed measures are tightly connected to each other to the point that both of them can be considered 'variazioni sul tema' of the same approach. In particular, $D_{k \eta}$ represents the dissimilarity of sample $k$ from the pooled set of species in the $N-1$ samples that differ from $k$. Therefore, this index, together with the 
corresponding beta diversity $\beta_{\eta}$, is directly related to the notion of originality (or distinctiveness,

203 Pavoine et al. 2017). A sample is functionally original if its functional characteristics are rare in the 204 pooled set of samples. The index is also related to the notion of the complementarity of a sample compared to a reference set of samples: complementarity being the gain in biodiversity units provided by adding an area (or sample) to a set of areas (samples) (Faith et al. 2004). These two notions (originality and complementarity) are used in conservation biology to identify sites with distinct species/functional/phylogenetic composition (and thus sites for which conservation actions should be a priority because of their distinct composition) (e.g. Mishler et al. 2014).

From the perspective of conservation biology, Kosman et al. (2019) recently proposed an additional indicator for estimating functional differences among samples: functional uniqueness, or singularity. Based on this approach, a sample that is on average quite distant from most samples but functionally similar to another sample has a lower conservation priority compared to a sample with the same average distance to other samples but without a close neighbor in functional space. To summarize this property, Violle et al. (2017) calculated the minimum pairwise distance between a focal sample and all other samples, while the singularity measure of Kosman et al. (2019) is based on variation in distances of the focal sample to all other samples, not just the nearest neighbor in trait space. Nonetheless, irrespective of how singularity is calculated, it can be easily derived from the distances $D_{h k}$ in Eq. 1 .

Unlike the vast majority of functional dissimilarity measures used in community ecology, the algorithmic index of Kosman (1996) and Gregorius et al. (2003), is not based on the excess of among-sample diversity compared to within-sample diversity (e.g. Chao et al. 2014; Chiu and Chao 2014; Pavoine and Ricotta 2014). Therefore, it is very flexible as it can be based on any betweenspecies dissimilarity measure of choice without restrictions on their geometrical properties (see e.g. Pavoine and Ricotta 2014). Also, the index of Kosman (1996) and Gregorius et al. (2003) satisfies an important requisite for functional dissimilarity measures which requires that dissimilarity remains unchanged if a given species $j$ is replaced by two functionally identical species with the same total abundance of $j$. For mathematical details, see Leinster and Cobbold (2012); Pavoine and Ricotta (2019). From an ecological viewpoint, this means that the measures that conform to this requisite summarize the functional dissimilarity among samples irrespective of the identity of the species that support these functions. Accordingly, this algorithmic dissimilarity is closer to the essence of functional dissimilarity than the measures that do not conform to this requisite.

Regarding the test for differences in functional beta diversity among different groups of samples, the principle is the same as that of Anderson (2006). However, the values of $D_{\bar{k}}$ and $D_{k \eta}$ are not influenced by the number of samples in each group. In addition, we do not need to calculate the 
236 functional centroid of each group, and this renders the test much easier to perform, especially if the 237 dissimilarities $D_{h k}$ and $D_{k \eta}$ are not embeddable in Euclidean space without distortion (for details, 238 see McArdle and Anderson 2001).

239 Nonetheless, alongside the pros, there are also a few potential cons for this test: like for the 240 Anderson test, the values of $D_{\bar{k}}$ and $D_{k \eta}$ are not fully independent of each other. This is because, 241 for a given sample $k$ the quantity $D_{\bar{k}}\left(D_{k \eta}\right)$ is obtained by averaging all dissimilarities $D_{h k}$ (all 242 species relative abundances $p_{j h}$ ) over all $N-1$ samples that are different from $k$ (see Eq. 3 and 4, 243 respectively). This nonindependence may become relevant for small numbers of samples such that 244 in the most critical situation of $N=2$, the values of $D_{\bar{k}}$ and $D_{k \eta}$ are identical for both samples.

245 Even more importantly, the randomization process associated to this test, while being 246 statistically sound, has only little biological foundation. Beta diversity describes the spatial 247 variability in species composition and is considered to be a key signature of a number of community 248 assembly processes, such as dispersal, habitat filtering, intra- and inter-specific competition, or the 249 species responses to environmental conditions (Bennet and Gilbert 2016). Therefore, while the 250 permutation of the dissimilarities $D_{\bar{k}}$ and $D_{k \eta}$ among sampling units has no clear biological 251 meaning, a biologically sound null model should provide some indication on whether differences in 252 beta diversity among groups of samples are actually related to deterministic assembly processes that 253 deviate from stochastic patterns of species co-occurrence (Chase et al. 2011). This may be achieved, 254 for example, by restricted permutation of species occurrences among the samples in each group. 255 However, to construct an adequate randomization test that correctly addresses the ecological 256 questions under study without confounding within group heterogeneity with between group 257 heterogeneity, some additional work is needed. In the meantime, the tests described in this paper 258 may represent an acceptable, though ecologically imperfect solution to the problem. 


\section{References}

Anderson, M.J. (2004) PERMDISP: a FORTRAN computer program for permutational analysis of multivariate dispersions (for any two-factor ANOVA design) using permutation tests. Department of Statistics, University of Auckland, New Zealand.

Anderson, M.J. (2006) Distance-based tests for homogeneity of multivariate dispersions. Biometrics 62: 245-253.

Anderson, M.J., Crist, T.O., Chase, J.M., Vellend, M., Inouye, B.D., Freestone, A.L., Sanders, N.J., Cornell, H.V., Comita, L.S., Davies, K.F., Harrison, S.P., Kraft, N.J.B., Stegen, J.C., Swenson, N.G. (2011) Navigating the multiple meanings of beta diversity: a road map for the practicing ecologist. Ecology Letters 14: 19-28.

Anderson, M.J., Ter Braak, C.T.F. (2003) Permutation tests for multi-factorial analysis of variance. Journal of Statistical Computation and Simulation 73: 85-113.

Bennet, J.R., Gilbert, B. (2016) Contrasting beta diversity among regions: how do classical and multivariate approaches compare? Global Ecology and Biogeography 25: 368-377:

Caccianiga, M., Luzzaro, A., Pierce, S., Ceriani, R. M., Cerabolini, B.E.L. (2006) The functional basis of a primary succession resolved by CSR classification. Oikos 112: 10-20.

Champely, S., Chessel, D. (2002) Measuring biological diversity using Euclidean metrics. Environmental and Ecological Statistics 9: 167-177.

Chao, A., Chiu, C.H. (2016) Bridging the variance and diversity decomposition approaches to beta diversity via similarity and differentiation measures. Methods in Ecology and Evolution 7: 919928.

Chao, A., Chiu, C.H., Jost, L. (2014) Unifying species diversity, phylogenetic diversity, functional diversity, and related similarity and differentiation measures through Hill numbers. Annual Review of Ecology, Evolution, and Systematics 45: 297-324.

Chao, A., Ricotta, C. (2019) Quantifying evenness and linking it to diversity, beta diversity, and similarity. Ecology 100: e02852.

Chase, J.M., Kraft, N.J.B, Smith, K.G., Vellend, M., Inouye, B.D. (2011) Using null models to disentangle variation in community dissimilarity from variation in $\alpha$-diversity. Ecosphere 2: art24. DOI:10.1890/ES10-00117.1.

Chiu, C.H., Chao, A. (2014) Distance-based functional diversity measures and their decomposition: a framework based on Hill numbers. PLOS ONE 9: e100014.

Dantzig, G.B., Thapa, M.N. (1997) Linear Programming. Springer, New York.

Diserud, O.H., Ødegaard, F. (2007) A multiple-site similarity measure. Biology Letters 3: 20-22.

Faith, D.P., Reid, C.A.M., Hunter, J. (2004) Integrating phylogenetic diversity, complementarity and endemism for conservation assessment. Conservation Biology 18: 255-261.

Gregorius, H.R., Gillet, E.M., Ziehe, M. (2003) Measuring differences of trait distributions between populations. Biometrical Journal 45: 959-973.

Hurlbert, S.H. (1971) The nonconcept of species diversity: a critique and alternative parameters. Ecology 52: 577-586.

Jost, L. (2007) Partitioning diversity into independent alpha and beta components. Ecology 88: 2427-2439.

Koleff, P., Gaston, K.J., Lennon, J.J. (2003) Measuring beta diversity for presence-absence data. Journal of Animal Ecology 72: 367-382.

Kosman, E. (1996). Difference and diversity of plant pathogen populations: a new approach for measuring. Phytopathology 86: 1152-1155.

Kosman, E. (2014) Measuring diversity: from individuals to populations. European Journal of Plant Pathology 138: 467-486.

Kosman, E., Burgio, K.R., Presley, S.J., Willig, M.R., Scheiner, S.M. (2019) Conservation prioritization based on trait-based metrics illustrated with global parrot distributions. Diversity and Distributions 25: 1156-1165. 
Kosman, E., Leonard, K.J. (2007). Conceptual analysis of methods applied to assessment of diversity within and distance between populations with asexual or mixed mode of reproduction. New Phytologist 174: 683-696.

\section{Supplementary Material}

Lande, R. (1996) Statistics and partitioning of species diversity, and similarity among multiple communities. Oikos 76: 5-13.

Legendre, P., De Cáceres, M. (2013) Beta diversity as the variance of community data: Dissimilarity coefficients and partitioning. Ecology Letters 16: 951-963.

Leinster, T., Cobbold, C.A. (2012) Measuring diversity: the importance of species similarity. Ecology 93: 477-489.

Levene, H. (1960) Robust tests for equality of variances. In: Olkin, I., Ghurye, S.G., Hoeffding, W., Madow, W.G., Mann, H.B. (Eds.) Contributions to probability and statistics. Stanford University Press, Stanford, pp. 278-292.

McArdle, B.H., Anderson, M.J. (2001) Fitting multivariate models to community data: a comment on distance-based redundancy analysis. Ecology 82: 290-297.

Mishler, B.D., Knerr, N., González-Orozco, C.E., Thornhill, A.H., Laffan, S.W., Miller, J.T. (2014) Phylogenetic measures of biodiversity and neo-and paleo-endemism in Australian Acacia. Nature Communications 5: 1-10.

Patil, G.P., Taillie, C. 1982. Diversity as a concept and its measurement. Journal of the American Statistical Association 77: 548-561.

Pavoine, S., Bonsall, M.B., Dupaix, A., Jacob, U., Ricotta, C. (2017) From phylogenetic to functional originality: guide through indices and new developments. Ecological Indicators 82: 196-205.

Pavoine, S., Ricotta, C. (2014) Functional and phylogenetic similarity among communities. Methods in Ecology and Evolution 5: 666-675.

Pavoine, S., Ricotta, C. (2019) Measuring functional dissimilarity among plots: Adapting old methods to new questions. Ecological Indicators 97: 67-72.

Ricotta, C. (2017) Of beta diversity, variance, evenness, and dissimilarity. Ecology and Evolution 7: 4835-4843.

Ricotta, C., Acosta, A.T.R., Caccianiga, M., Cerabolini, B.E.L., Godefroid, S., Carboni, M. (2020) From abundance-based to functional-based indicator species. Ecological Indicators 118: 106761.

Ricotta, C., de Bello, F., Moretti, M., Caccianiga, M., Cerabolini, B.E.L., Pavoine, S. (2016). Measuring the functional redundancy of biological communities: A quantitative guide. Methods in Ecology and Evolution 7: 1386-1395.

Ricotta, C., Kosman, E., Laroche, F., Pavoine, S. (2021). Beta redundancy for functional ecology. Methods in Ecology and Evolution 12: 1062-1069.

Tuomisto, H. (2010a) A diversity of beta diversities: straightening up a concept gone awry. Part 1. Defining beta diversity as a function of alpha and gamma diversity. Ecography 33: 2-22.

Tuomisto, H. (2010b) A diversity of beta diversities: straightening up a concept gone awry. Part 2. Quantifying beta diversity and related phenomena. Ecography 33: 23-45.

Violle, C., Thuiller, W., Mouquet, N., Munoz, F., Kraft, N.J.B., Cadotte, M.W., Livingstone, S.W., Mouillot, D. (2017). Functional rarity: The ecology of outliers. Trends in Ecology \& Evolution, 32: 356-367.

Whittaker, R. (1972) Evolution and measurement of species diversity. Taxon 21: 213-251.

Appendix 1. R scripts and examples for the calculation of the two functional beta diversity measures proposed in this paper

Appendix 2. R scripts in text format 
359 Author contributions. CR conceived the idea; CR, EK and SP developed the methodology; MC and 360 BC collected the data; all authors analyzed the data; CR took the lead in writing the main text. SP 361 wrote the R script. All authors revised the manuscript critically and approved the final version.

Conflicts of Interest. The authors declare that they have no known competing financial interests or personal relationships that could have appeared to influence the work reported in this paper.

Funding. This research received no external funding. 
367 Figure 1. Box plots of the beta diversity components (dissimilarity coefficients) $D_{\bar{k}}$ and $D_{k \eta}$ for the 368 three successional stages of the Alpine vegetation of the Rutor glacier. ESS = early-successional 369 stage; MSS = mid-successional stage; LSS = late-successional stage. Different letters a and b 370 indicate significantly different distributions at $\mathrm{p}<0.001$ for $D_{\bar{k}}$ and $\mathrm{p}<0.01$ for $D_{k \eta}$ 371 (permutational t-test with Holm adjustment for multiple tests based on 9999 randomizations).

372

373

374

375

376

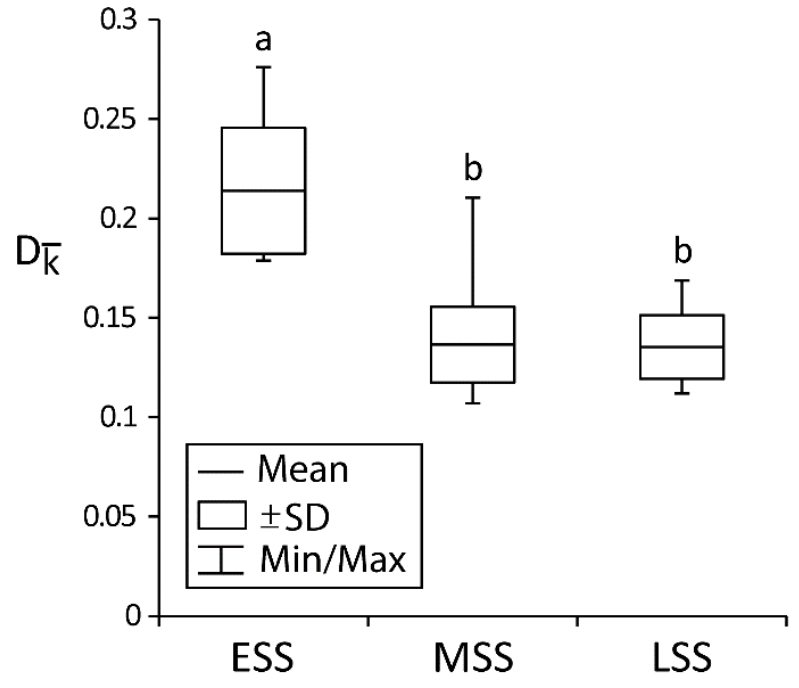

